\title{
Early Postnatal Development in the Rat is Characterized by Accumulation of Highly Unsaturated Triacylglycerols
}

\author{
ZHEN-YU CHEN AND STEPHEN C. CUNNANE \\ Department of Nutritional Sciences, Faculty of Medicine, University of Toronto, \\ Toronto, Ontario, Canada MSS 1 A8
}

\begin{abstract}
Our objective was to determine the quantitative changes of individual triacylglyercol (TG) species in liver and carcass during early postnatal growth. Pregnant rats were killed at $\mathrm{d} 21$ of pregnancy, and neonatal rats were killed at $\mathbf{d} 3$ or 9 after birth. Quantitative changes in fatty acids and TG species of fetal/neonatal liver and carcass were determined using capillary gas liquid chromatography. At postnatal d 3 or 9 compared with fetal d 21, total carcass TG increased 11- to 12-fold, with nonessential fatty acids increasing 8- to 9-fold, n-6 essential fatty acids (EFA) increasing 34- to 44-fold, and n-3 EFA increasing 19- to 29-fold, respectively. Total neonatal liver TG increased 13-fold from fetal d 21 to postnatal d 3 , with a 6-fold increase in non-EFA, a 34-fold increase in total $n-6$ EFA and a 65-fold increase in total n-3 EFA. At postnatal d 3 compared with fetal d 21, larger molecular weight liver TG classes (C56-C64) increased 68-fold, followed by lower molecular weight $\mathrm{TG}$ classes $\mathrm{C40}-\mathrm{C} 48$ (19-fold) with only a 6-fold increase in C50-C54. In liver, highly unsaturated TG classes (C56-C64) accounted for $49 \%$ of total TG at postnatal d 3 and consisted mainly of arachidonic, docosahexaenoic, and linoleic acids accompanied by palmitic and oleic acids. During early postnatal development, TG species containing one, two, or even three 20-22 EFA may be structurally important themselves or serve as direct substrates for synthesis of phospholipids. The unique accumulation of C54-C64 TG species, especially in liver, indicates that highly unsaturated TG are a quantitatively important EFA pool during early life. (Pediatr Res 31: 4751, 1992)
\end{abstract}

Abbreviations

$\mathrm{P}$, palmitic acid (16:0)

$\mathrm{S}$, stearic acid (18:0)

O, oleic acid (18:1n-9)

L, linoleic acid (18:2n-6)

LCFA, long-chain fatty acids

TG, triacylglycerol

EFA, essential fatty acids

The traditional approach to determine the EFA required by a developing fetus or newborn involves examination of the fatty acid composition of breast milk or determination of fetal and

Received April 30, 1991; accepted August 8, 1991.

Correspondence and reprint requests: Dr. Zhen-Yu Chen, Department of Nutritional Sciences, Faculty of Medicine, University of Toronto, Toronto, Ontario, Canada M5S 1 A8.

Supported by the Natural Science and Engineering Research Council of Canada and the Ontario Ministry of Health. neonatal composition and accretion of EFA at different stages of growth (1). Therefore, it was suggested that the milk formula for low birth weight human infants should be one corresponding to that of the baby's body itself (2). Formula milk TG with a stereospecific structure and fatty acid composition similar to that of breast milk have been reported to be absorbed best (1). Many studies have addressed the changes in LCFA composition and metabolism that occur during fetal and neonatal development (2-11), and, although the postnatal changes in EFA composition, especially in $\mathrm{TG}$, are dramatic $(12,13)$, their speciation has not been previously described. Such information may lead to insight regarding structural versus energy-providing functions of EFA such as linoleic acid (18:2n-6).

The development of the capillary column coated with a polarizable phenylmethylsilicone phase has made it possible to resolve TG species by carbon number and unsaturation (14). Recently, the potential application of this column in the analysis of plasma TG profiles has been reported (15), and the results are most encouraging, although overlap of TG species with same total carbon number and double bond number still occurs. We have recently shown that this column also resolves $\mathrm{TG}$ species of rat tissue lipids and that linoleoyl-enriched TG species increase in maternal liver toward term (16) and are also responsive to fasting (17).

We report here that the composition of TG species in the liver and carcass changes markedly during the first postnatal week, becoming temporarily very complex through the accumulation of both medium-chain TG and highly unsaturated TG species in comparison to the situation prenatally or after the first week of life.

\section{MATERIALS AND METHODS}

Animals. Second parity female Sprague-Dawley rats (Charles River Canada, St. Constant, Quebec, Canada) were housed individually in suspended, stainless steel, wire-bottomed cages in the animal facilities of the Faculty of Medicine, University of Toronto. They were housed, fed, and handled according to the guidelines of Canadian Council on Animal Care. The rats were fed a rodent Chow diet (Ralston-Purina Co., St. Louis, MO) and tap water throughout pregnancy and lactation. The Chow contained $4.5 \%$ fat by weight with $18: 2 n-6$ and $\alpha$-linolenic acid $(18: 3 n-3)$ at 27 and $3 \%$ of total fatty acids, respectively. The female rats were mated with experienced males, and vaginal plugs were taken as a sign of successful conception (d 1 of pregnancy). The rats were killed on $d 21$ of pregnancy or $d+3$ or +9 of lactation $(n=4 /$ date; + denotes lactation period). For d-21 fetal samples, pregnant rats were anesthetized under carbon dioxide gas, their abdomens were opened, and whole fetal carcasses were collected, followed by decapitation of the fetuses and removal of their livers. Neonatal rats at $d+3$ and +9 were also killed by decapitation after anesthesia under carbon dioxide. In 
Table 1. Fatty acid composition of fetal and neonatal carcass and liver $T G(\mu \mathrm{g} / \mathrm{g}$, mean $\pm S D ; n=4 /$ group $)$

\begin{tabular}{|c|c|c|c|}
\hline & \multirow[b]{2}{*}{ Fetal (d 21) } & \multicolumn{2}{|c|}{ Neonatal } \\
\hline & & $d+3$ & $\mathrm{~d}+9$ \\
\hline \multicolumn{4}{|c|}{ Carcass fatty acids } \\
\hline $16: 0$ & $1563 \pm 36$ & $10566 \pm 499$ & $11587 \pm 273 \dagger$ \\
\hline $16: 1 \mathrm{n}-7$ & $198 \pm 42$ & $1388 \pm 192 *$ & $923 \pm 102 \dagger$ \\
\hline $18: 0$ & $277 \pm 19$ & $2545 \pm 154^{*}$ & $2385 \pm 38$ \\
\hline $18: 1 \mathrm{n}-9$ & $686 \pm 49$ & $11152 \pm 461^{*}$ & $9639 \pm 301 \dagger$ \\
\hline $18: 2 n-6$ & $143 \pm 43$ & $7404 \pm 231^{*}$ & $6631 \pm 240 \dagger$ \\
\hline $20: 3 n-6$ & $6 \pm 3$ & $540 \pm 115^{*}$ & $273 \pm 34$ \\
\hline $20: 4 n-6$ & $55 \pm 18$ & $1658 \pm 269^{*}$ & $786 \pm 102 \dagger$ \\
\hline $22: 4 n-6$ & $18 \pm 3$ & $424 \pm 35^{*}$ & $205 \pm 68 \dagger$ \\
\hline $22: 5 n-6$ & $9 \pm 6$ & $116 \pm 76^{*}$ & $34 \pm 24 \dagger$ \\
\hline $18: 3 n-3$ & $3 \pm 2$ & $347 \pm 38^{*}$ & $376 \pm 70$ \\
\hline $20: 5 n-3$ & $3 \pm 2$ & $308 \pm 56^{*}$ & $273 \pm 34$ \\
\hline $22: 5 n-3$ & $9 \pm 6$ & $617 \pm 76^{*}$ & $308 \pm 68 \dagger$ \\
\hline $22: 6 n-3$ & $79 \pm 33$ & $1465 \pm 384^{*}$ & $820 \pm 34 \dagger$ \\
\hline \multicolumn{4}{|c|}{ Liver fatty acids } \\
\hline $16: 0$ & $762 \pm 64$ & $7921 \pm 1137^{*}$ & $2800 \pm 580 \dagger$ \\
\hline $16: 1 \mathrm{n}-7$ & $214 \pm 42$ & $590 \pm 168^{*}$ & $97 \pm 10 \dagger$ \\
\hline $18: 0$ & $230 \pm 80$ & $1264 \pm 169^{*}$ & $486 \pm 29 \dagger$ \\
\hline $18: \ln -9$ & $1405 \pm 186$ & $6278 \pm 253^{*}$ & $1848 \pm 107 \dagger$ \\
\hline $18: 2 n-6$ & $233 \pm 80$ & $6826 \pm 253^{*}$ & $1750 \pm 190 \dagger$ \\
\hline $20: 3 n-6$ & $26 \pm 3$ & $632 \pm 126^{*}$ & $117 \pm 10 \dagger$ \\
\hline $20: 4 n-6$ & $99 \pm 19$ & $4761 \pm 126^{*}$ & $690 \pm 39 \dagger$ \\
\hline $22: 4 n-6$ & $19 \pm 3$ & $1053 \pm 168^{*}$ & $165 \pm 10 \dagger$ \\
\hline $22: 5 n-6$ & $22 \pm 6$ & $421 \pm 84^{*}$ & $39 \pm 9 \dagger$ \\
\hline $18: 3 n-3$ & $6 \pm 3$ & $253 \pm 42^{*}$ & $68 \pm 19 \dagger$ \\
\hline $20: 5 n-3$ & $32 \pm 25$ & $1348 \pm 253^{*}$ & $233 \pm 9 \dagger$ \\
\hline $22: 5 n-3$ & $10 \pm 6$ & $2149 \pm 234^{*}$ & $398 \pm 19 \dagger$ \\
\hline $22: 6 n-3$ & $144 \pm 61$ & $8679 \pm 1601^{*}$ & $1040 \pm 42 \dagger$ \\
\hline
\end{tabular}

$* p<0.01$ compared with $\mathrm{d} 21$.

$\dagger p<0.01$ compared with $\mathrm{d}+3$.

each case, the livers and carcasses were washed in saline, blotted, weighed, placed immediately in chloroform containing $0.02 \%$ wt/vol of butylated hydroxytoluene (Sigma Chemical Co., St. Louis, MO), and stored at $-20^{\circ} \mathrm{C}$ until analyzed.

Fatty acid and $T G$ species analysis. All organic solvents used in the lipid extraction or chromatography were glass-redistilled before use. Methanol was added to the samples stored in chloroform to a final concentration of $2: 1(\mathrm{vol} / \mathrm{vol})$ with a solventto-tissue sample ratio of 10:1. Samples were then homogenized at full speed using a Brinkmann Polytron (Brinkmann Instruments, Rexdale, Ontario, Canada), followed by addition of $0.9 \%$ saline and then centrifugation. The organic phase was pipetted and evaporated to a constant lipid weight under nitrogen gas. The total tissue TG in each sample was separated by thin-layer chromatography using a solvent mixture (hexane:diethyl ether:glacial acetic acid, 80:20:1) and transmethylated using 14\% boron trifluoride in methanol (Sigma Chemical Co.) at $90^{\circ} \mathrm{C}$ under nitrogen gas for $30 \mathrm{~min}$. Fatty acid profiles of the total TG were determined by gas liquid chromatography using a capillary column (Durabond $225,30 \mathrm{~m} \times 25 \mu \mathrm{m}$, inner diameter; JW Scientific Inc. Folsom, CA) in a gas liquid chromatograph (HP 5890 A; Hewlett-Packard Co. Palo Alto, CA) with automated sample delivery, injection, and peak integration (17).

An aliquot of each total lipid extract was used for isolation of the total TG with addition of triheptadecanoin as an internal standard for quantification. A second thin-layer chromatography separation was used for this purpose, after which the TG species were analyzed using a Varian 3700 gas chromatograph equipped with a TG column $(25 \times 25 \mu \mathrm{m}$, inner diameter, coated with 10 $\mu \mathrm{m}$ of $65 \%$ phenylmethyl silicone film; Quadrex, New Haven, CT) operating at 320 to $355^{\circ} \mathrm{C}$, as previously described (17).
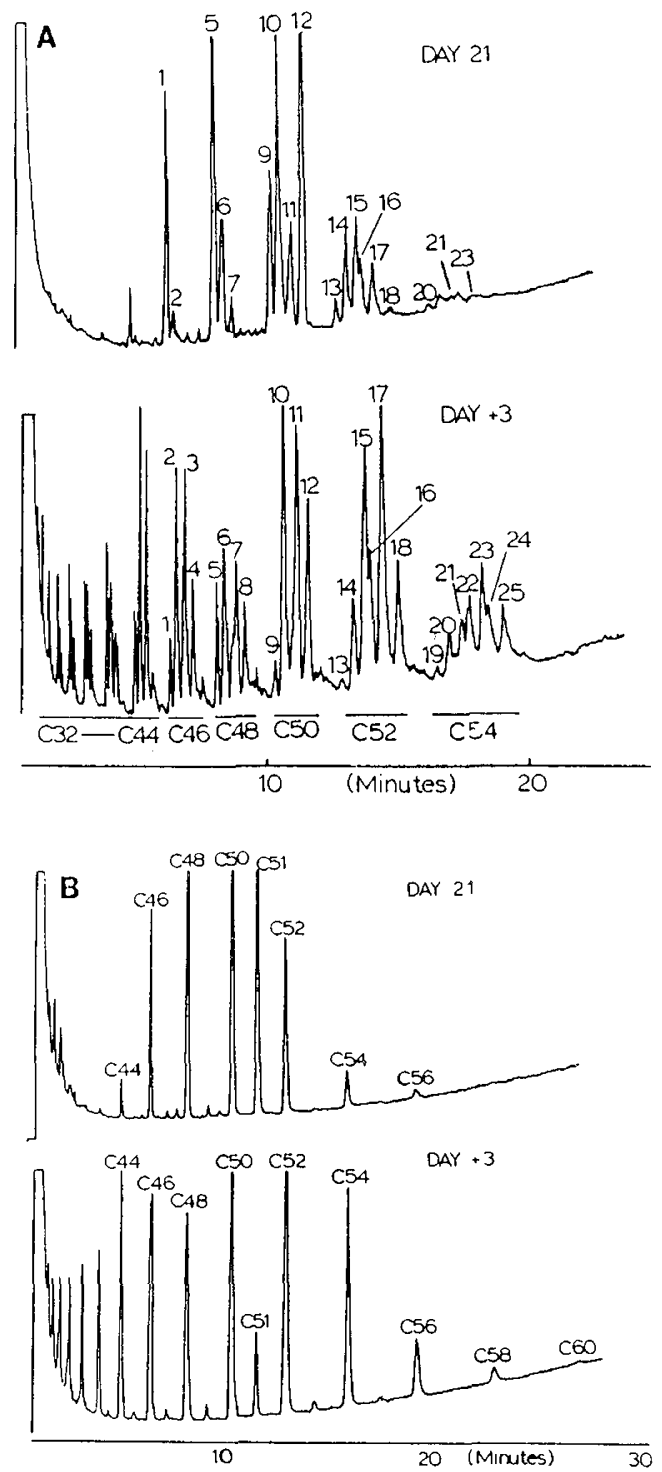

Fig. 1. Gas liquid chromatograms of carcass TG species from a fetal rat (top, d 21) or a neonatal rat (bottom, $\mathrm{d}+3$ ). Column: fused silica capillary $(25 \mathrm{~m} \times 0.25 \mathrm{~mm}$, inner diameter) coated with $65 \%$ phenylmethyl silicone; temperature: $320-355^{\circ} \mathrm{C}$ at $2^{\circ} \mathrm{C} / \mathrm{min}$; carrier gas: hydrogen ( 25 pounds per square inch); and detector: flame ionization detector. $A$, unhydrogenated TG. $B$, hydrogenated TG. Peak identifications (+ possible alternative peaks) where $M$ is myristic acid, Po is palmitoleic acid, Mo is myristoleic acid, and La is lauric acid: $1, \mathrm{MPP} ; 2, \mathrm{MPPo}(+$ MMO); 3, MPoPo (+ MoPPo + MML); 4, MoPoPo (+ MoML); 5, PPP (+ MSP); 6, PPPo (+ MPO); 7, PPoPo (+ LaOO + MPL); 8, PoPoPo (+ $\mathrm{LaOL}+\mathrm{MPOL}) ; 9, \mathrm{PSP}(+\mathrm{MSP}) ; 10, \mathrm{POP}(+\mathrm{MSP}) ; 11, \mathrm{PLP}$ (+ PoOP + MOO); 12, internal standard (triheptadecanoin); 13, PSS; 14, PSO (+ PoSS); 15, POO; 16, PSL; 17, POL (+ PoOO); 18, PLL; 19, SSO; 20, SOO (+ SSL); 21, OOO; 22, SLO; 23, OLO; 24, SLL; and 25, OLL.

Designation of TG species resolved by gas liquid chromatography is by carbon number. Thus, $\mathrm{C} 54$ is a $\mathrm{TG}$ with predominantly three 18-carbon fatty acids or one 16-carbon, one 18-carbon, and one 20 -carbon fatty acid. The individual species are identified by the combination of the first letter of the fatty acids involved, but this is not stereospecific, e.g. PLL $=$ LPL $=$ LLP. Recovery of TG species greater than C56 decreased with increasing unsaturation. TG species were therefore compared across time, but interspecies comparisons were not made. In addition, hydrogenation permitted high recovery but loss of resolution of C20-22 fatty acid-containing TG species (17).

Statistics. Analysis of variance followed by $t$ test (two-tailed) was used for statistical evaluation of differences between groups. 

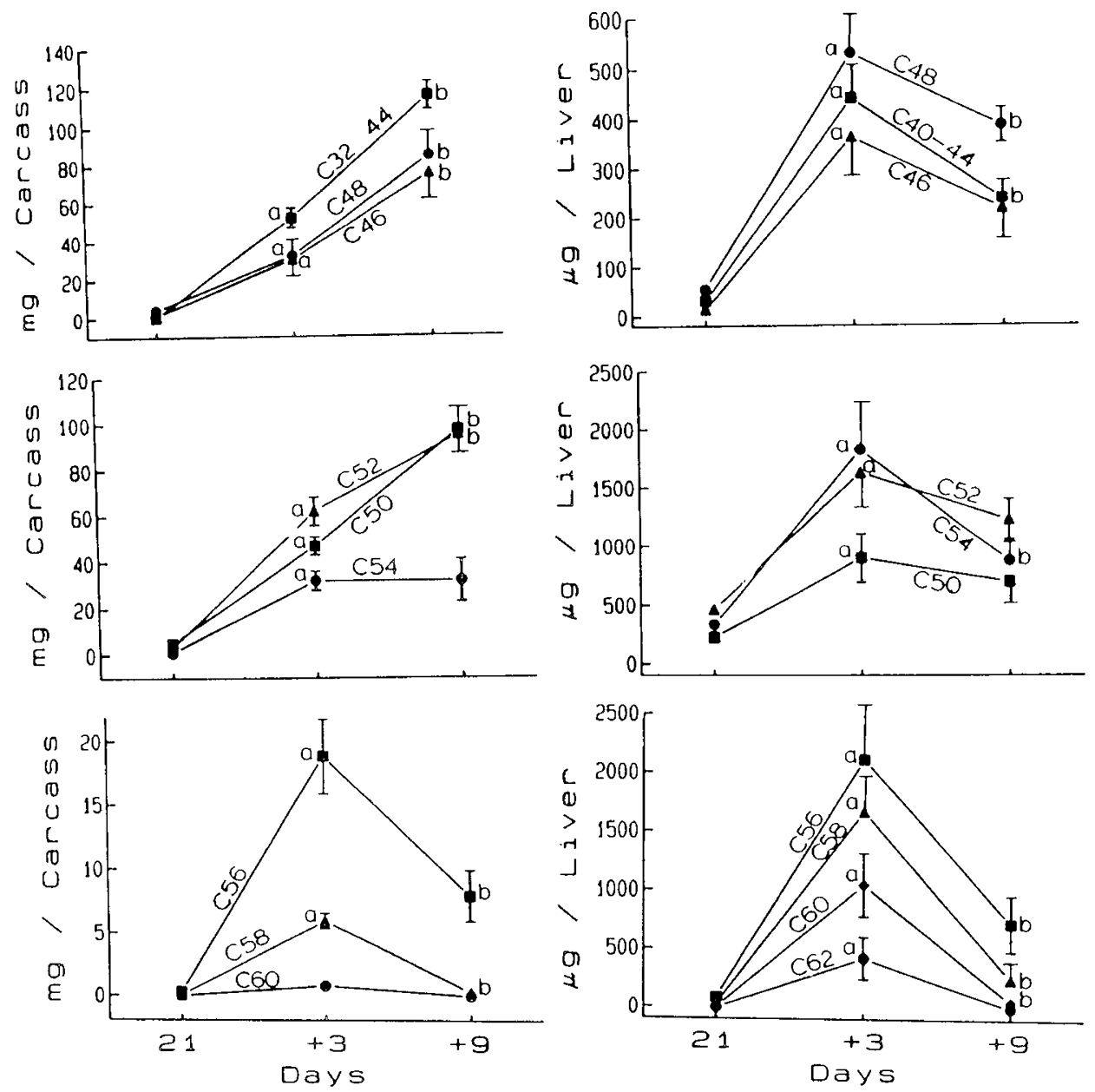

Fig. 2. Quantitative changes in both neonatal carcass and liver TG subclasses during late prenatal ( $\mathrm{d} 21)$ and early postnatal development ( +3 and $\mathrm{d}+9) .{ }^{a} p<0.01$ in contrast to $\mathrm{d} 21 ;{ }^{b} p<0.01$ in contrast to $\mathrm{d}+3$.

\section{RESULTS}

Carcass and liver fatty acids. Postnatally $(\mathrm{d}+3$ or +9 , compared with d 21), total neonatal carcass TG quantitatively increased 11 - to 12-fold, with non-EFA increasing 8-to 9-fold, n6 EFA increasing 34- to 44-fold, and n-3 EFA increasing 19- to 29 -fold, respectively. In relation to d 21 , total neonatal liver TG quantitatively increased 13 -fold at $\mathrm{d}+3$ of lactation, with a $6-$ fold increase in non-EFA, a 34-fold increase in n-6 EFA, and a 65-fold increase in n-3 EFA. Among n-6 EFA, 18:2n-6 and $20: 4 n-6$ increased the most from d 21 to +3 (29- and 48-fold, respectively). Within n-3 EFA, 18:3n-3, 20:5n-3, 22:5n-3, and 22:6n-3 increased 42-, 42-, 215-, and 60-fold, respectively. Compared with $d+3$, total neonatal liver $T G$ decreased at $d+9$ by $77 \%$, with non-EFA, n-6 EFA, and n-3 EFA decreasing, by 67 , 80 , and $86 \%$, respectively, but still remaining significantly higher than at $\mathrm{d} 21$ (Table 1).

Carcass TG species. C56-60 TG species (containing at least one 20-22 fatty acid) were not resolved in the unhydrogenated samples (Fig. $1 A$ ), but were resolved as saturated TG after hydrogenation (Fig. $1 B$ ). The neonatal rat had a carcass $\mathrm{TG}$ species profile distinctly different from that of the fetal rat at $\mathrm{d}$ 21. At $\mathrm{d}+3$, the neonatal carcass contained $\mathrm{C} 32-\mathrm{C} 44 \mathrm{TG}$ subclasses that were undetectable at $\mathrm{d} 21$. Compared with $\mathrm{d} 21$, all carcass TG species from C32 to C58 were increased significantly by $\mathrm{d}+3$, contributing a 12 -fold increase in total TG. However, at $\mathrm{d}+9, \mathrm{C} 32-\mathrm{C} 48$ were still as high or higher than at $\mathrm{d}+3$, but C56-C60 were decreased in comparison with $d+3$ (Fig. 2).

At d 21, 16:0-enriched TG species [PPP, PSP, POP, PPPo (Po $=$ palmitoleic acid), and PLP] dominated the carcass TG species profile (Table 2), which is in agreement with the carcass TG fatty acid data indicating that 16:0 accounted for $49 \%$ of total fatty acids at $d 21$ (Table 1). On the other hand, 18:2n-6-enriched TG species were quantitatively minor in the carcass TG at this time. Between $\mathrm{d} 21$ and $\mathrm{d}+3$, the total of dilinoleoyl TG species (OLL, SLL, and PLL) increased 61-fold; the total of monolinoleoyl TG species (OLO, SLO, PLO, PSL, and PLP) increased 19-fold, whereas the total of other nonlinoleoyl-containing TG species increased 6-fold. In the carcass, accumulation of C56-C60 TG classes containing 20-22 EFA was also greatest at $\mathrm{d}+3$ (5-fold increase over $d 21)$. However, at $d+9$, total carcass $T G$ decreased by $21 \%$ compared with $\mathrm{d}+3$, and most individual TG species $(\mu \mathrm{g} / \mathrm{g})$ were decreased correspondingly (Table 2, Fig. 2).

Liver TG species. Based on total acyl carbon number, all liver TG subclasses were increased significantly at $\mathrm{d}+3$ in contrast to prenatal values (Fig. 2 and 3, Table 3). Larger molecular weight TG classes (C56-C62) in the liver collectively increased 68-fold, followed by lower molecular weight TG classes (C40-C48, 19fold increase). The intermediate $\mathrm{TG}$ class (C50-C54; mainly consisting of 16:0, 18:1n-9, 18:2n-6, and 18:0) increased 6-fold. From d 21 to +3 , dilinoleoyl TG species in the liver (OLL, SLL, and PLL) increased 52-fold, monolinoleoyl TG species (OLO, SLO, PLO, PSL, and PLP) increased 4-fold, and nonlinoleoyl TG species $(C<54)$ increased 5 -fold. In contrast to $d+3$, at $d+9$ all TG species in neonatal liver were decreased $(\mu \mathrm{g} / \mathrm{g})$, contributing to a $77 \%$ decrease in total neonatal liver TG (Fig. 2, Table 3).

From the liver TG fatty acid data at $d+3$ (Table 1 ), many species could contribute to C56, but fewer possibilities are likely for C58-C64 (Fig. 3): the TG species within C58 would include 
Table 2. Composition of fetal/neonatal carcass $T G$ species $(\mu \mathrm{g} / \mathrm{g}, \text { mean } / n=4)^{*}$

\begin{tabular}{lccc} 
& & \multicolumn{2}{c}{ Neonatal } \\
\cline { 3 - 4 } TG species $\dagger$ & Fetal $(\mathrm{d} 21)$ & $\mathrm{d}+3$ & $\mathrm{~d}+9$ \\
\hline Nonlinoleoyl & & & \\
MPP & $248 \pm 18$ & $417 \pm 46 \ddagger$ & $889 \pm 55 \S$ \\
MPPo & $40 \pm 12$ & $1465 \pm 85 \ddagger$ & $2177 \pm 126 \S$ \\
MPoPo & $\mathrm{ND}$ & $1700 \pm 131$ & $1850 \pm 208$ \\
MoPoPo & $\mathrm{ND}$ & $737 \pm 100$ & $427 \pm 109 \S$ \\
PPP & $591 \pm 61$ & $717 \pm 34 \ddagger$ & $833 \pm 170$ \\
PPPo & $290 \pm 60$ & $1158 \pm 277 \S$ & $2162 \pm 41 \S$ \\
PPoPo & $55 \pm 16$ & $1798 \pm 88 \ddagger$ & $2137 \pm 106 \S$ \\
PoPoPo & ND & $1000 \pm 119$ & $810 \pm 109$ \\
PSP & $228 \pm 46$ & $299 \pm 42$ & $283 \pm 102$ \\
POP & $561 \pm 17$ & $3122 \pm 280 \ddagger$ & $3174 \pm 68$ \\
PSS & $72 \pm 16$ & $138 \pm 42 \ddagger$ & $111 \pm 20$ \\
PSO & $181 \pm 13$ & $862 \pm 157 \ddagger$ & $753 \pm 55$ \\
POO & $145 \pm 37$ & $1968 \pm 167 \ddagger$ & $1750 \pm 51$ \\
SSO & ND & $45 \pm 7$ & $104 \pm 27 \S$ \\
SOO & $50 \pm 10$ & $457 \pm 42 \ddagger$ & $140 \pm 27 \S$ \\
OOO & $33 \pm 15$ & $542 \pm 81 \ddagger$ & $151 \pm 40 \S$ \\
Monolinoleoyl & & & \\
PLP & $231 \pm 57$ & $3438 \pm 404 \ddagger$ & $3364 \pm 136$ \\
PSL & $73 \pm 15$ & $984 \pm 123 \ddagger$ & $875 \pm 143$ \\
POL & $112 \pm 16$ & $3733 \pm 419 \ddagger$ & $2363 \pm 58 \S$ \\
SLO & $67 \pm 10$ & $866 \pm 169 \ddagger$ & $312 \pm 109 \S$ \\
OLO & $43 \pm 5$ & $1032 \pm 215 \ddagger \mathrm{a}$ & $606 \pm 228 \S$ \\
Dilinoleoyl & & & \\
PLL & $48 \pm 17$ & $1271 \pm 135 \ddagger$ & $814 \pm 38 \S$ \\
SLL & ND & $866 \pm 146$ & $323 \pm 75 \S$ \\
OLL & ND & $814 \pm 180$ & $606 \pm 157 \S$ \\
\hline ND & & &
\end{tabular}

* ND, undetectable $(<1 \mu \mathrm{g} / \mathrm{g})$; Mo, myristoleic acid; and Po, palmitoleic acid.

$\dagger$ Only the main species are shown; others may also be present that overlap some of those shown.

$\ddagger p<0.01$ compared with $\mathrm{d} 21$.

$\S p<0.01$ compared with $\mathrm{d}+3$.

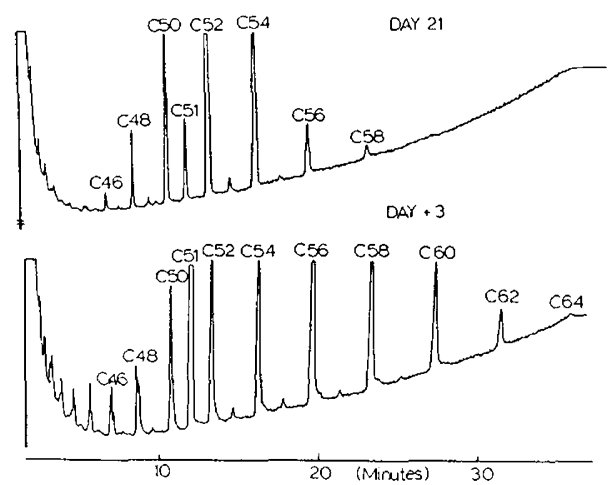

Fig. 3. Gas liquid chromatograms of hydrogenated liver TG species from a fetal (top, d 21) or neonatal rat (bottom, $\mathrm{d}+3)$. See Figure 1 for conditions.

oleoyl linoleoyl docosahexaenoyl glycerol, palmitoyl arachidonoyl docosahexaenoyl glycerol, oleoyl diarachidonoyl glycerol, or linoleoyl diarachidonoyl glycerol; within C60 the main TG species would be oleoyl arachidonoyl docosahexaenoyl glycerol, palmitoyl didocosahexaenoyl glycerol, and linoleoyl arachidonoyl docosahexaenoyl glycerol; the TG species within $\mathrm{C} 62$ would be mainly diarachidonyl docosahexaenoyl glycerol, linoleoyl didocosahexaenoyl glycerol, and oleoyl didocosahexaenoyl glycerol; and the TG species within C64 would be mainly arachidonoyl didocosahexaenoyl glycerol. Their specific identification is not possible at present because of a lack of standards as well as methods for accurate separation and identification of TG species with more than 60 acyl carbons.
Table 3. Composition of fetal/neonatal liver $T G$ species $(\mu \mathrm{g} / \mathrm{g}$, mean $\pm S D / n=4$ )*

\begin{tabular}{lccc}
\hline & & \multicolumn{2}{c}{ Neonatal } \\
\cline { 3 - 4 } TG species $\dagger$ & Fetal $(\mathrm{d} 21)$ & $\mathrm{d}+3$ & $\mathrm{~d}+9$ \\
\hline Nonlinoleoyl & & & \\
PPP & $26 \pm 5$ & $681 \pm 58 \ddagger$ & $216 \pm 21 \S$ \\
PPPo & $84 \pm 3$ & $783 \pm 133 \ddagger$ & $352 \pm 26 \S$ \\
PPoPo & $37 \pm 3$ & $646 \pm 142 \ddagger$ & $250 \pm 30 \S$ \\
PSP & $46 \pm 7$ & $349 \pm 89 \ddagger$ & $222 \pm 42 \S$ \\
POP & $223 \pm 36$ & $2141 \pm 424 \ddagger$ & $678 \pm 61 \S$ \\
PSS & $45 \pm 13$ & $429 \pm 88 \ddagger$ & $255 \pm 50 \S$ \\
PSO & $100 \pm 19$ & $411 \pm 31 \ddagger$ & $230 \pm 19 \S$ \\
POO & $569 \pm 89$ & $1384 \pm 407 \ddagger$ & $497 \pm 82 \S$ \\
SSO & $48 \pm 4$ & $133 \pm 49 \ddagger$ & $49 \pm 29 \S$ \\
SOO & $249 \pm 10$ & $482 \pm 150 \ddagger$ & $117 \pm 45 \S$ \\
OOO & $241 \pm 19$ & $1349 \pm 535 \ddagger$ & $247 \pm 21 \S$ \\
Monolinoleoyl & & & \\
PLP & $322 \pm 32$ & $1119 \pm 146 \ddagger$ & $586 \pm 20 \S$ \\
PSL & $284 \pm 68$ & $690 \pm 199 \ddagger$ & $248 \pm 53 \S$ \\
POL & $250 \pm 17$ & $2504 \pm 287 \ddagger$ & $966 \pm 90 \S$ \\
SLO & $326 \pm 76$ & $1296 \pm 217 \ddagger$ & $312 \pm 26 \S$ \\
OLO & $163 \pm 23$ & $518 \pm 248 \ddagger$ & $178 \pm 44 \S$ \\
Dilinoleoyl & & & \\
PLL & ND & $1106 \pm 75$ & $414 \pm 25 \S$ \\
SLL & $88 \pm 39$ & $2287 \pm 438 \ddagger$ & $572 \pm 128 \S$ \\
OLL & ND & $1251 \pm 332$ & $383 \pm 96 \S$ \\
\hline ND & &
\end{tabular}

* ND, undetectable $(<1 \mu \mathrm{g} / \mathrm{g})$; and Po, palmitoleic acid.

† Only the main species are shown; others may also be present that overlap some of those shown.

$\ddagger p<0.01$ compared with $\mathrm{d} 21$.

$\S p<0.01$ compared with $\mathrm{d}+3$.

\section{DISCUSSION}

In the present study, it is evident that a marked but transitory increase in the quantitative content of LCFA and TG species of carcass and liver occurs during early postnatal development in the rat. The increase began at $\mathrm{d} 21$ (final prenatal day) and peaked at $d+3$ (postnatal), but was markedly reduced by $d+9$. Although these data are affected by changes in carcass and liver weight pre- to postnatally, their expression as either $\mu \mathrm{g} / \mathrm{g}$ or $\mu \mathrm{g} /$ carcass (liver) does not affect the overall interpretation. These quantitative changes in TG composition are not evident from the proportional fatty acid composition on these days, which, to our knowledge, is the only form in which fatty acid data $(2-13)$ or TG subclass data (17) on this topic have previously been published.

In neonatal carcass and liver, TG containing a high proportion of $n-6$ and n-3 EFA were characteristically accumulated during the first week of life (around $d+3$ ). Among n-6 EFA in the total TG pool, 18:2n-6 and 20:4n-6 were accumulated most, whereas within n-3 EFA the most accumulated were $22: 5 n-3$ and $22: 6 n-$ 3. The quantitative accumulation of large amounts of $20-22$ carbon EFA in the TG pool seems to be unique to early postnatal life, inasmuch as they are usually components of tissue phospholipids during adulthood.

Prenatally, total carcass had a lower TG content $(3.2 \mathrm{mg} / \mathrm{g}$ at d 21) than it did postnatally $(40.5-35.9 \mathrm{mg} / \mathrm{g}$ between $\mathrm{d}+3$ and +9 ). Postnatally, the TG structure appeared to be more complicated than it did prenatally by including lower molecular weight TG species C32-C44 $(0.3-1.0 \mathrm{mg} / \mathrm{g})$, which are characteristic of those in the milk $(3,11,18)$. These data suggest that the fatty acids in carcass TG originate from both milk fatty acids and de novo synthesis in the neonate. Because milk TG are readily hydrolyzed in the stomach and small intestine of the neonatal rat $(18-21)$, the presence of TG species $<C 40$ in the neonatal liver suggests that they are rapidly synthesized and stored in both neonatal rat liver and carcass. In maternal liver $T G, 18: 2 n-6$, $20: 4 n-6$, and 22:6n-3 also increased significantly throughout late 
pregnancy to the first $3 \mathrm{~d}$ of lactation (10), suggesting that these EFA are accumulated in the maternal liver TG before being transferred to the mammary gland, where they become available to the neonate during lactation.

The 18:2n-6-containing TG species are the only polyunsaturated TG species that we can clearly identify using this methodology. These species range from $\mathrm{C} 46$ to $\mathrm{C} 54$. In each case, before birth, the species containing $18: 2 n-6$ were minor relative to those not containing 18:2n-6. Postnatally, the 18:2n-6-containing species in both carcass and liver increased markedly, frequently from undetectable levels at d 21: dilinoleoyl-TG species (PLL, OLL, and SLL) increased the most, whereas monolinoleoyl and nonlinoleoyl TG species increased considerably less. Thus, there seems to be important acyl transferase specificity favoring the incorporation of 18:2n-6 into neonatal carcass and liver TG during early postnatal development. The significance of this marked accumulation of 18:2n-6-enriched TG species in carcass and liver is not clear from the present study. In view of the preference to oxidize 18:2n-6 over other LCFA (22), 18:2n-6 accumulation in specific liver and carcass $T G$ species may be important as an energy source, possibly for thermoregulation during early life. Additionally, such 18:2n-6-enriched TG species may have structural or precursor function, although this has not yet been described.

In the liver, the accumulation of TG species containing 20-22 EFA (C56-C64) was greatest at $d+3$, with a significant decrease occurring by $\mathrm{d}+9$. Part of the decrease $(\mu \mathrm{g} / \mathrm{g})$ was due to an increase in both liver and carcass weight between $d+3$ and +9 . Nevertheless, even expressed as $\mu \mathrm{g} /$ liver (or carcass), the data clearly indicate the transitory nature of the rapid postnatal increase in highly unsaturated TG that occurs postnatally. These TG species were unusual compared with those in adult rats (17) and may themselves have specific structural functions. Alternatively, they may serve as direct substrates for synthesis of phospholipids, e.g. oleoyl arachidonoyl docosahexaenoyl, which, after removal of the oleoyl moiety via hydrolysis, could be directly converted to arachidonoyl docosahexaenoyl diacylglycerol. This may be an efficient way to supply EFA for cellular regulation and development during rapid growth.

TG is quantitatively minor in brain, but its metabolism may play an important role via acyl exchange with phospholipids in the CNS during early postnatal development (9). Although brain TG is not addressed in the present study, the polyunsaturated fatty acids accumulated in liver TG may be sources of EFA for rapid postnatal development of brain and other organs. Two distinct changes in $20: 4 n-6$ and 22:6n-3 were observed: 1 ) qualitively, 20:4n-6 and 22:6n-3 account for 11 and $21 \%$, respectively, of total liver TG fatty acids at $\mathrm{d}+3$, whereas in adult rat they account for 3 and $5 \%$, respectively (10); and 2) quantitatively, 20:4n-6 and 22:6n-3 increased 48- and 60-fold, respectively, from prenatal $\mathrm{d} 21$ to postnatal $\mathrm{d}+3$. This accumulation of $20: 4 n-6$ and $22: 6 n-3$ in liver TG may be partially due to de novo synthesis from their corresponding precursors, $18: 2 n-6$ and $18: 3 n-3$, via desaturation and elongation or due to uptake from milk lipids. Thus, liver TG serves as transitory store of these polyunsaturated fatty acids, which may be transferred to brain and other organs when rapid growth occurs during the first week of life. Therefore, the structural characteristics and metabolism of the large molecular TG species (C58-C64) containing 20:4n-6 and 22:6n-3 accumulated in liver early in postnatal life need further investigation.

\section{REFERENCES}

1. Clandinin MT, Chappel JE, Van Aerde JEE 1989 Requirements of newborn infants for long chain polyunsaturated fatty acids. Acta Paediatr Scand 351:63-71

2. Widdowson EM, Southgate DAT, Hey EN 1979 Body composition of the fetus infant. In: Visser HKA (ed) Proceeding of Fifth Nutricia Symposium on Nutrition and Metabolism of Fetus and Infants. Martinus Nijhoff, The Hague, Holland, pp 169-177

3. Sinclair AJ 1974 Fatty acid composition of liver lipids during development of rat. Lipids 9:809-818

4. Crawford MA, Hassam AG, Rivers JPW 1978 Essential fatty acids and the vulnerability of artery during growth. Postgrad Med J 54:149-153

5. Ravel D, Chambaz J, Pepin D, Manier M-C, Bereziat G 1985 Essential fatty acid interconversion during gestation in the rats. Biochim Biophys Acta $883: 161-164$

6. Sinclair AJ 1975 Incorporation of radioactive polyunsaturated fatty acid into liver and brain of developing rat. Lipids 10:175-184

7. Clandinin MJ, Chappell JE, Heim T, Sawyer PR, Chance PW 1981 Fatty acid utilization in perinatal de novo synthesis of tissue. Early Hum Dev 5:355366

8. Bourre JM, Piciott M, Dumont O, Pascal G, Durand G 1990 Dietary linoleic acid and polyunsaturated fatty acids in rat brain and other organs. Minimal requirements of linoleic acid. Lipids 25:465-472

9. Cook HW 1981 Metabolism of triacylglycerol in developing rat brain. Neurochem Res 6:1217-1229

10. Cunnane SC, Armstrong JK 1990 Long chain fatty acid composition of maternal liver lipids during pregnancy and lactation in the rat: comparison of triglyceride to phospholipid. J Nutr 120:338-345

11. Sinclair AJ, Crawford MA 1973 The effect of a low-fat maternal diet on neonatal rats. Br J Nutr 29:127-132

12. Cunnane SC, Chen ZY 1991 Triacylglycerol: an important pool of essential fatty acids during early postnatal development in the rat. Am J Physiol (in press)

13. Cunnane SC, Chen ZY 1991 Quantitative changes in long chain fatty acids during fetal and early postnatal development in the rat. Am $\mathbf{J}$ Physiol (in press)

14. Geeraert E, Sandra P 1985 Capillary GC of triglycerides in fats and oils using a high temperature phenylmethylsilicone stationary phase, part I. J High Resol Chromatogr Chromatogr Commun 8:415-422

15. Kuksis A, Myher JJ, Sandra P 1990 Gas-liquid chromatographic profiling of plasma lipid using high-temperature-polarizable capillary column. J Chromatogr 500:427-444

16. Chen ZY, Yang J, Menard CR, Cunnane SC 1991 Linoleoyl-enriched triacylglycerol species increase in maternal liver during late pregnancy in the rat. Lipids (in press)

17. Chen ZY, Cunnane SC 1991 Short-term energy deficit causes net accumulation of linoleoyl-enriched triacylglycerols in rat liver. FEBS Lett 280.393-396

18. Staggers JE, Fernando-Warnakulasuriya GJP, Wells MA 1981 Studies on fat digestion, absorption, and transport in the suckling rat. II. Triacylglycerols: molecular species, stereospecific analysis, and specificity of hydrolysis by lingual lipase. J Lipid Res 22:675-679

19. Aw TY, Grigor MR 1980 Digestion and absorption of milk triacylglycerols in 14-day-old suckling rats. J Nutr 110:2133-2140

20. Fernando-Warnakulasuriya GJP, Staggers JE, Frost SC, Wells MA 1981 Studies on fat digestion, absorption, and transport in the suckling rat. I. Fatty acid composition and concentrations of major lipid components. J Lipid Res 22:668-674

21. Bitman J, Wood DL, Liao TH, Fink CS, Hamosh P, Hamosh M 1985 Gastric lipolysis of milk lipids in suckling rats. Biochim Biophys Acta 834:58-64

22. Leyton J, Drury PJ, Crawford MA 1987 Different oxidation of saturated and unsaturated fatty acids in vivo in the rat. Br J Nutr 57:383-393 